\title{
Cognitive control of language production in bilinguals involves a partly independent process within the domain-general cognitive control network: Evidence from task-switching and electrical brain activity
}

\author{
David A. Magezi ${ }^{\mathrm{a}, 1}$, Asaid Khateb ${ }^{\mathrm{b}, \mathrm{c}}$, Michael Mouthon ${ }^{\mathrm{a}, \mathrm{b}}$, Lucas Spierer ${ }^{\mathrm{a}}$, Jean-Marie Annoni ${ }^{\mathrm{a}, \mathrm{b}, \mathrm{d}, *}$ \\ a Neurology Unit, Department of Medicine, Faculty of Sciences, University of Fribourg, Fribourg, Switzerland \\ ${ }^{\mathrm{b}}$ Laboratory of Experimental Neuropsychology, Department of Neurology, University Hospital and Faculty of Medicine, Geneva, Switzerland \\ ${ }^{\mathrm{c}}$ Edmond J. Safra Brain Research Center for the Study of Learning Disabilities, Department of Learning Disabilities, Faculty of Education, University of Haifa, Israel \\ ${ }^{\mathrm{d}}$ Neurology Unit, Hospital of Fribourg, Fribourg, Switzerland
}

\begin{abstract}
In highly proficient, early bilinguals, behavioural studies of the cost of switching language or task suggest qualitative differences between language control and domain-general cognitive control. By contrast, several neuroimaging studies have shown an overlap of the brain areas involved in language control and domain-general cognitive control. The current study measured both behavioural responses and eventrelated potentials (ERPs) from bilinguals who performed picture naming in single- or mixed-language contexts, as well as an alphanumeric categorisation task in single- or mixed-task context. Analysis of switch costs during the mixed-context conditions showed qualitative differences between language control and domain-general cognitive control. A $2 \times 2$ ANOVA of the ERPs, with domain (linguistic, alphanumeric) and context (single, mixed) as within-participant factors, revealed a significant interaction, which also suggests a partly independent language-control mechanism. Source estimations revealed the neural basis of this mechanism to be in bilateral frontal-temporal areas.
\end{abstract}

\section{Introduction}

Bilingual and multilingual speakers have the remarkable ability to speak in a single language, or to quickly and accurately change between languages in a bi- or multilingual context (Grosjean, 1998). The nature of the cognitive processes underlying this bilingual language control is still a matter of debate. Although there is general agreement that bilingual language control involves domain-general cognitive control, two important questions remain: Is bilingual language control completely mediated by the domain-general cognitive-control system, or does an independent language-specific cognitive control mechanism exist? If so, what is its neural substrate? The aim of the current study was to address these questions by combining behavioural and neuroimaging approaches.

The behavioural paradigm employed was picture-naming in a mixed-language context. Bilingual participants named pictures in either their first (L1) or second language (L2), depending on a

\footnotetext{
* Corresponding author at: Neurology Unit, Department of Medicine, Faculty o Sciences, University of Fribourg, Ch. du Musée 5, CH 1700 Fribourg, Switzerland. Fax: +41264268135.

E-mail address: jean-marie.annoni@unifr.ch (J.-M. Annoni).

1 Present address: Donders Institute for Brain, Cognition, and Behaviour, Radboud University, Nijmegen, The Netherlands.
}

visual cue, and responses to trials were divided into two groups. Trials in which the naming was performed in a different language to the preceding trial are referred to as "switch" trials. In contrast, "repeat" trials, are those where the language is the same as for the preceding trial. Reaction times have been shown to be slower for switch than for repeat trials, and this is known as a "switch cost". In late "unbalanced" bilinguals, who are more highly proficient in L1 than L2, there is an asymmetric switch cost, that is, a greater switch cost for L1 (the "easier" language) than for L2 (e.g. Meuter \& Allport, 1999). Models of bilingual language control posit that this asymmetric switch cost is due to the difference in difficulty between the two languages (see Section 4). Importantly, an asymmetric switch cost can also be seen in switching between two tasks of differing difficulty, where there is a greater switch cost for the easier task. This qualitative similarity in switch costs supports the hypothesis that language control, in late bilinguals, is mediated by the same mechanism that mediates domain-general cognitive control (including task-switching). At first glance, it appears that this hypothesis is also supported by data from bilinguals who acquired both L1 and L2 from an early age and are highly proficient in both. These early, high-proficient, balanced bilinguals show a symmetric switching cost; that is, the switch cost is the same for L1 and L2, which are both equally "easy". However, when switching between L1 and a much weaker L3, these same early 
bilinguals also show a symmetric switch cost. Recently, Calabria, Hernández, Branzi, and Costa (2012) recruited a group of early, high-proficient bilinguals to perform not only picture naming in a mixed-language condition, but also task-selection in a mixedtask condition (card sorting by either colour or shape). Although switch costs were symmetric in all the mixed-language conditions (L1-L2 or L1-L3), they were asymmetric in the mixed-task condition. Costa and colleagues (2004) and Calabria et al., (2012), propose that these studies provide evidence for a language control mechanism in highly-proficient, early bilinguals that is at least partly independent from the domain-general cognitive control system. As in the study of Calabria et al. (2012), participants in the current study performed both picture naming in a mixed-language context, and task selection in a mixed-task context (alphanumeric categorisation in L1). Unlike Calabria et al. (2012), the participants of the current study were more heterogeneous in terms of age of acquisition of L2, language proficiency and languages spoken in order to examine whether the behavioural evidence for a partlyindependent language control mechanism can also be found in a different population of bilinguals.

The neural basis of the hypothesised partly-independent, language-specific control mechanism remains unclear. For this reason, the current study measured the electrical brain activity (electroencephalography, EEG) of the bilingual participants. Non-invasive neuroimaging techniques such as functional magnetic neuroimaging (fMRI) and EEG have greatly contributed to our understanding of the neural basis of language control in bilinguals (e.g., Abutalebi, Della Rosa, Tettamanti, Green, \& Cappa, 2011; Abutalebi et al., 2008; Jackson, Swainson, Mullin, Cunnington, \& Jackson, 2001; Jackson, Swainson, Mullin, Cunnington, \& Jackson, 2004; Khateb et al., 2007; Price, Green, \& Von Sutdnitz, 1999; for review, see Abutalebi \& Green, 2008; Luk, Green, Abutalebi, \& Grady, 2011). Most of these studies have compared the brain responses of bilinguals performing identical or very similar tasks (e.g. picture naming) in a mixed-language context and in a single-language (monolingual) context, and brain activity has been shown to be higher for the mixed-language context in a cortical-subcortical network of brain regions that is bilateral, but predominantly left-lateralized, and includes inferior and middle frontal gyrus, precentral gyrus, supplementary motor area (SMA), caudate, anterior cingulate cortex (ACC), and supramarginal gyrus (SMG). It has been proposed that this predominantly frontal cortical-subcortical network plays a crucial role in bilingual language control (Abutalebi \& Green, 2008; Luk et al., 2011), and further support of this hypothesis has come from studies of bilingual aphasia (e.g., Aglioti \& Fabbro, 1993; Abutalebi, Della Rosa, Tettamanti, Green, \& Cappa, 2009; Abutalebi, Miozzo, \& Cappa, 2000; Kho et al., 2007), intracranial stimulation (e.g., Moritz-Gasser \& Duffau, 2009), and transcranial magnetic stimulation studies (for review, see Hervais-Adelman, Moser-Mercer, \& Golestani, 2011). However, it is known that most of the areas in this cortical-subcortical network are also involved in task switching (e.g. Dove, Pollmann, Schubert, Wiggins, \& von Cramon, 2000; Kimberg, Aguirre, \& D'Esposito, 2000) and in general executive control (for review, see Ye \& Zhou, 2009). To explore the overlap between bilingual control and domain-general cognitive control, some studies have measured brain activity not only during linguistic conditions (single- and mixed-language contexts), but also during task selection conditions: that is, the same participants also perform either a single task (single-task context), or select one of two tasks depending on a cue (mixed-task context; Abutalebi et al., 2008; Abutalebi et al., 2011b; Khateb et al., 2007). An important limitation of the EEG study of Khateb et al. (2007) and the fMRI study of Abutalebi et al. (2008) is that the mixed-task condition was linguistically complex; participants had to name a picture or generate a verb related to the picture (both in L1). Therefore these tasks were closely related to the mixed-language condition (picture naming in
L1 or L2). In order to examine the extent of overlap between bilingual language control and domain-general cognitive control mechanisms, it is important to measure brain responses related to bilingual language control as well as those related to domain-general cognitive control using a different, non-linguistic domain. Recently, such a study was performed by Abutalebi et al.'s (2011). Event-related fMRI data was collected while bilingual participants performed not only picture naming in a mixed-language context, but also a flanker task, where trials were neutral, congruent or incongruent to asses conflict monitoring. Consistent with previous research, it was found that both language control and domaingeneral cognitive control (conflict monitoring) engaged the dorsal ACC (see also Section 4). In contrast to Abutalebi et al.'s (2011) study, the current study also included a single-language context. This allows inclusion of brain activity related to both "sustained" and "transient" language control during the single- and mixedlanguage contexts, respectively (Christoffels, Firk, \& Schiller, 2007; Wang, Kuhl, Chen, \& Dong, 2009).

In the current study, the EEG data were subjected to a twoby-two repeated-measures analysis of variance (ANOVA) using the within-participant factors of domain (linguistic, alphanumeric) and context (single, mixed). The result of the interaction between these two factors is crucial for our examination of the overlap between language and domain-general control mechanisms. On the one hand, the presence of a significant interaction would strongly suggest a difference between the brain activity associated with language selection and that of general task selection, and we can perform source reconstruction to estimate the brain regions responsible for the interaction. This may suggest that language control relies on the same brain areas as domain-general cognitive control, but with different levels of activity; or it may provide evidence for language control modules outside of this domain-general network. On the other hand, the absence of any significant interaction would suggest that, with the current paradigm, the same brain regions are involved in both language selection and general task selection, to the same extent.

\section{Methods}

\subsection{Participants}

The presented data are from 26 female bilingual students (mean age $=22.8$ years, age range $19-29$ years) at the School of Translation and Interpretation (ETI) of the University of Geneva. In order to maximise differences in second language (L2) proficiency (see below), 12 participants were recruited from the first year of the ETI undergraduate program ("Bachelor"), and 14 were recruited from the first year of the ETI postgraduate program ("Master"). As expected, the post-graduate students were on average 4.0 years older, [independent $t$-test, $t(24)=5.19, p<0.001$ ]. A variety of first and second languages (L1-L2 pairs) were included in the current study: French-English (9 participants), French-German (7), German-English (5), and German-French (5). According to the Edinburgh Inventory (Oldfield, 1971), all 26 participants were strongly right-handed (laterality quotient, LQ > 57). Although data were collected from 36 bilingual students, the data of four participants had to be excluded due to excessive artefacts in the EEG data (more than about $1 / 3$ of trials included peristimulus eye blinks). A further six participants were excluded because they differed from the majority of the group in two features which are thought to be important in language processing: gender ( 1 male), and handedness ( 2 ambidextrous and 3 strongly left-handed). All participants had normal or corrected-to-normal vision, and none presented a history of psychiatric or neurological disease. Written informed consent was obtained from all participants, but in order not to bias 
responses, participants were not made aware of the purposes of the study. The experimental procedures were in accordance with the Code of Ethics of the World Medical Association (Declaration of Helsinki) and were approved by the Ethics Committee of the University of Geneva Medical School.

\subsection{Assessment of language proficiency and exposure}

The mean age of acquisition of L2 was from $11.3 \pm 0.5$ years. Unfortunately, due to schedule conflicts, not all participants were able to take part in all tests of language proficiency and exposure. 23 participants were able to take part in the formal L2 proficiency assessment, during which they were presented a passage of about 1000 words in their L1. The task was to summarise the entire text into L2, as well as to translate a portion (about 200 words) into L2. The answers of each participant were scored by two independent language experts, and the final proficiency score was the mean of the experts' scores. 25 participants were able to perform an untimed picture naming task in L2, based on a set of 40 images from a standard naming test (DO-80; Metz-Lutz et al., 1991). In order to assess the amount of exposure to languages, 22 participants also completed a questionnaire (Wartenburger et al., 2003), with sections on media (TV and radio), family, friends, girlfriends/boyfriends, university (classmates and teaching), reading (newspapers and books) and recreational activities (hobbies, sports, music).

\subsection{Stimuli and experimental procedure}

Visual stimuli were presented on a screen, which was divided into two halves by a horizontal line (Fig. 1). There were four experimental conditions.

Alphanumeric tasks: In the first condition, referred to as "alphanumeric single" (ANSin) the visual stimulus was a letter-digit pair such as " $9 \mathrm{~A}$ " and the participant had to perform a "single" task, that is, state whether the letter was a vowel or a consonant. The letter-digit pairs comprised of one digit between 2 and 9 (inclusive), and either a vowel (A, E, I, U) or a consonant (B, G, M, R). The within-pair orders (e.g. "4B" or "B4") were counterbalanced. For each participant, the letter-digit pair was always presented in the same half of the screen, that is, either above or below the horizontal line, throughout the entire first condition (ANSin), and this position was counterbalanced across participants. In the second condition, referred to as "alphanumeric mixed" (ANMix), the visual stimuli were also of letter-digit pairs, but this time there were two possible tasks. The letter-digit pair could appear in either part of the screen, and when the letter-digit pair was in the same half of the screen as for the ANSin condition, the task was the same as in the ANSin condition; that is, to categorise the letter as vowel or consonant. In contrast, when the stimulus appeared in the opposite half of the screen, the task was to categorise the digit as either odd or even. These alphanumeric tasks and spatial cueing are an adaptation of Rogers and Monsell's (1995) paradigm.

Linguistic tasks: The same spatial cue was used in the third and fourth conditions, where instead of letter-number pairs, the stimuli were line drawings of objects from the compiled database of Alario and Ferrand (1999). In the third condition, referred to as "linguistic single" (LiSin), the objects were always presented in the same half of the screen and participants had to name the objects in their first language (L1). Finally, in the fourth condition, referred to as "linguistic mixed" (LiMix), the participants had to name the object in either their first (L1) or second language (L2) depending on which half of the screen it appeared in. For this condition, particular attention was paid to avoid images eliciting cognate names, for example, "Apfel" and "apple" in German and English.

48 different letter-digit pair conditions were generated for the ANSin condition. A further 96 pairs were generated for the ANMix condition. In the same way, the LiSin and LiMix conditions comprised 48 and 96 different line drawings, respectively. Each letter-digit pair or line-drawing was only presented once to each participant. Trials were blocked into runs of 48 trials (approximately 4 min duration), with 1 min break between runs. Within each run, the experimental condition (ANSin, ANMix, LiSin or LiMix) was kept constant, and the order of trials was randomized. The conditions were presented in the following order: ANSin (1 run), followed by ANMix ( 2 runs), LiSin (1 run), and finally, LiMix ( 2 runs). This order was chosen to prevent the participants from being in "bilingual" mode (Grosjean 1998; Grosjean 2001) during the first three conditions which were performed only in L1. Note that the single and mixed conditions comprised one and two runs, respectively. This was done in order to present an equal number (48) of letter (alphanumeric) or L1 (linguistic) trials in the single and mixed conditions.

To start each trial, a central fixation cross was presented for $500 \mathrm{~ms}$. Next, the visual stimulus was presented for $300 \mathrm{~ms}$ immediately followed by a blank screen. The duration of the blank screen was variable (randomly chosen from $2350,2750,3150$ or $3950 \mathrm{~ms}$ ) resulting in a mean trial duration of 3950 ms. Participants were asked to make all responses as fast and as accurately as possible. Stimulus delivery was with E-Prime (Psychology Software Tools, Sharpsburg, PA). The visual stimuli and horizontal line were presented in black on a white background (refresh rate $70 \mathrm{~Hz}$ ) $130 \mathrm{~cm}$ away from the participants at a visual angle of $2-3^{\circ}$. Verbal responses were recorded using a digital minidisc recorder.
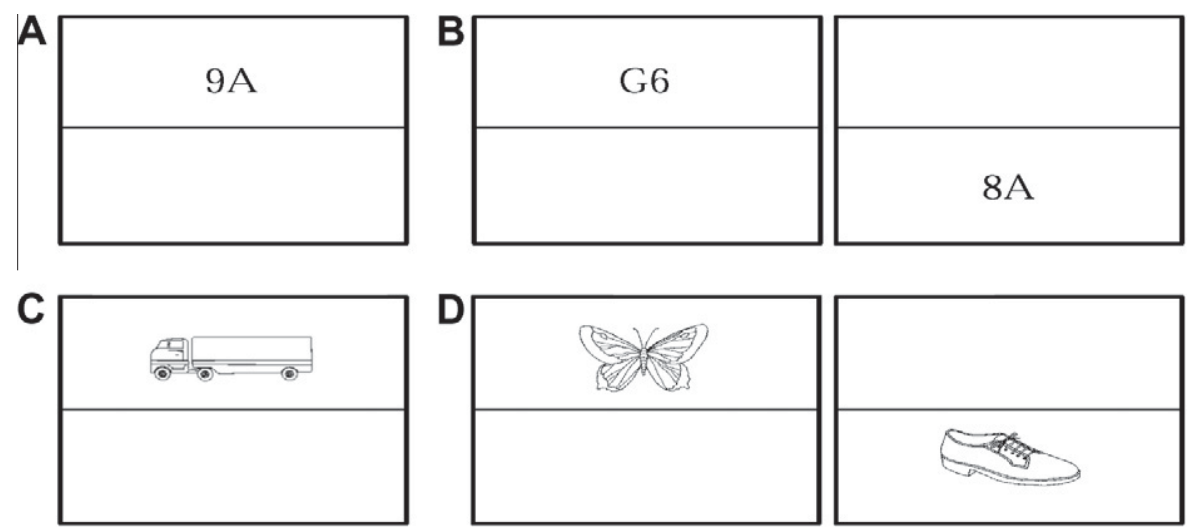

Fig. 1. Examples of stimuli used in the four experimental conditions: (A) Alphanumeric single (ANSin), (B) alphanumeric mixed (ANMix), (C) linguistic single (LiSin), and (D) linguistic mixed (LiMix). See Section 2.3 for details. 


\subsection{EEG recording and data analysis}

The EEG was continuously recorded with a sampling rate of $500 \mathrm{~Hz}$ (with online lowpass filtering at $200 \mathrm{~Hz}$ ) from 204 electrodes (256-channel system from Electrical Geodesic, Inc., Oregan, USA). The ground and reference electrodes were placed at the inion and vertex ("Cz"), respectively.

Preprocessing of raw data was performed using the EEGLAB toolbox (Delorme \& Makeig, 2004), which runs under Matlab, and Cartool (Brunet, Murray, \& Michel, 2011). The data were (1) bandpass filtered between 0.1 and $30 \mathrm{~Hz}$ using a -48 -dB per octave, zerophase filter (8th-order Butterworth filter applied in both forward and reverse time order), (2) bandstop filtered (45-55 Hz) to remove electrical line noise, (3) divided into stimulus-locked epochs covering the period from $-100 \mathrm{~ms}$ to $500 \mathrm{~ms}$ relative to stimulus onset and (4) baseline-corrected for the entire period. In the mixed conditions, only the letter (vowel-consonant task in ANMix) and first language (L1 in LiMix) stimuli were considered. This was done because these were present in both the single and mixed conditions, thereby permitting a comparison across contexts (e.g., see Khateb et al., 2007). Only epochs associated with correct behavioural responses were entered for further analysis. Epochs with amplitude changes exceeding $100 \mu \mathrm{V}$ in any electrode were rejected. The average number of epochs accepted for the four conditions was $43.1 \%, 44.9 \%$, $43.1 \%$, and $42.2 \%$ for ANSin (alphanumeric single), ANMix (alphanumeric mixed), LiSin (linguistic single) and LiMix (linguistic mixed), respectively. For each recording block, artefact electrodes, when present (on average fewer than 0.7 electrodes per participant), were interpolated using a 3D-spline algorithm. For further processing, ERPs were interpolated onto a 128-electrode montage.

Each time-point of the resulting ERPs was subject to a $2 \times 2$ repeated-measures ANOVA, with domain (alphanumeric, linguistic) and context (single, mixed) as within-participant factors. This was performed on each of the 128 electrodes.

\subsection{Source estimation}

The sources in the brain were estimated using a distributed linear inverse solution and the local autoregressive average (LAURA) regularization approach (Grave de Peralta, Gonzalez Andino, \& Gomez Gonzalez, 2004; Grave de Peralta, Gonzalez Andino, Lantz, Michel, \& Landis, 2001). This approach takes into account the biophysical behaviour of electric fields; that is, activity at one point depends on the activity at neighbouring points according to electromagnetic laws. The solution space comprised 3005 nodes distributed throughout the grey matter of the average brain of the Montreal Neurological Institute (MNI; courtesy of Grave de Peralta Menendez and Gonzalez Andino, University Hospital of Geneva, Geneva, Switzerland). As with the ERP data, the source-estimate data was subject to a time-wise $2 \times 2$ ANOVA with the same within-participant factors (domain and context), and similarly, this was performed for each node ("solution point").

Visual inspection of the results of the node-wise ANOVA revealed time-periods during which there were significant main effects or interactions (see Figs. 4 and 5). To show which solution points were responsible for the results (interaction or main effect), separate ANOVAs were performed for the average activity over these periods of interest, and the relevant results $(p<0.001)$ displayed on axial MRI slices of the MNI brain.

\section{Results}

\subsection{Proficiency test and language exposure}

Scores in the formal L2 proficiency test were positively correlated with accuracy in the untimed picture naming test in L2 $(r=0.44, p=0.036)$ as well as with age of participant $(r=0.71$, $p<0.001)$. L2 proficiency was not significantly correlated with age of acquisition of $\mathrm{L} 2(r=0.12, p=0.587)$. The responses to the language exposure questionnaire indicated that the participants were exposed for $4.2 \pm 0.8 \mathrm{~h} /$ day more to L1 than to $\mathrm{L} 2$ (paired $t$ test: $t(21)=5.00, p<0.001)$.

\subsection{Behavioural results: analysis of switch costs}

In the ANMix condition the mean reaction times were $1023 \pm 30,1177 \pm 38,1027 \pm 39,1093 \pm 31$ ms for number-repeat, number-switch, letter-repeat, and letter-switch trials, respectively (Table 1, Fig. 2C). A repeated measures ANOVA with the factors of task (letter or number categorisation) and switch status (repeat, switch) resulted in significant main effects of task $[F(1,25)=23.05, \quad p<0.001]$, switch status $[F(1,25)=43.30$, $p<0.001]$, and a significant interaction $[F(1,25)=5.10, p=0.033]$. Planned comparisons confirmed that the switch cost (difference in reaction times between switch and repeat trials) was greater for the number than for the letter task (paired $t$-test: $t(25)=2.26$, $p=0.033$ ). In the LiMix condition, mean reaction times were $1006 \pm 26,1042 \pm 29,1300 \pm 51$, and $1288 \pm 42 \mathrm{~ms}$ for L1-repeat, L1-switch, L2-repeat, and L2-switch respectively (Table 1, Fig. 2D). A repeated measures ANOVA with the factors of language (L1, L2) and switch status showed a significant main effect of language $[F(1,25)=63.19, p<0.001]$. There was no significant main effect of switch status $[F(1,25)=0.30, p=0.590]$, and no significant interaction $[F(1,25)=1.48, p=0.236]$. This shows that the switch cost was non-significant and was about the same for both L1 and L2, which was confirmed by planned comparisons (paired $t$-test: $t(25)=1.21, p=0.236)$.

Calabria et al. (2012) found that the direction of switch cost asymmetry varied as a function of experimental block for a mixed-language, but not a mixed-task condition. To examine whether this was also the case in the current study, two separate ANOVAs were performed on the reaction-times with the withinparticipant factors of task (letter or number) for ANMix or language (L1 or L2) for LiMix, switch status, and block (first or second). For the ANMix condition, there was no significant effect of block $[F(1,25)=0.815, p=0.375]$. Although there was a trend towards a significant interaction between block and switch status $[F(1,25)=3.43, p=0.076]$, there were no significant interactions involving the factor of block [block $*$ task: $F(1,25)=0.08$, $p=0.781$; three-way: $F(1,25)=0.21, p=0.886]$. As expected, there were significant main effects of switch status $[F(1,25)=43.30$, $p<0.001]$ and task $[F(1,25)=23.046, p<0.001]$, and a significant switch status $*$ task interaction $[F(1,25)=5.096, p=0.033]$. Similarly, for the linguistic conditions, there was no significant effect of block $[F(1,25)=1.67, p=0.208]$. Again, there was a trend towards a significant block * switch-status interaction $[F(1,25)=3.269, p=0.083]$ and no significant interactions involving the block factor [block $*$ language interaction: $F(1,25)=0.43$, $p=0.520$; three-way: $F(1,25)=0.033, p=0.857]$. As expected, there was a significant main effect of language $[F(1,25)=63.194$,

\section{Table 1}

Reaction times (in ms, \pm standard error of the mean) in the mixed-context conditions, as a function of domain, task/language and switch status. Switch cost is the difference in reaction time between repeat and switch trials. ANMix: alphanumeric mixed, LiMix: alphanumeric mixed.

\begin{tabular}{llllr}
\hline Domain & Task/language & Repeat & Switch & Switch cost \\
\hline ANMix & Letter & $1027 \pm 39$ & $1093 \pm 31$ & $66 \pm 28$ \\
& Number & $1023 \pm 30$ & $1177 \pm 38$ & $154 \pm 23$ \\
LiMix & L1 & $1006 \pm 26$ & $1042 \pm 29$ & $35 \pm 14$ \\
& L2 & $1300 \pm 51$ & $1288 \pm 42$ & $-12 \pm 38$ \\
\hline
\end{tabular}



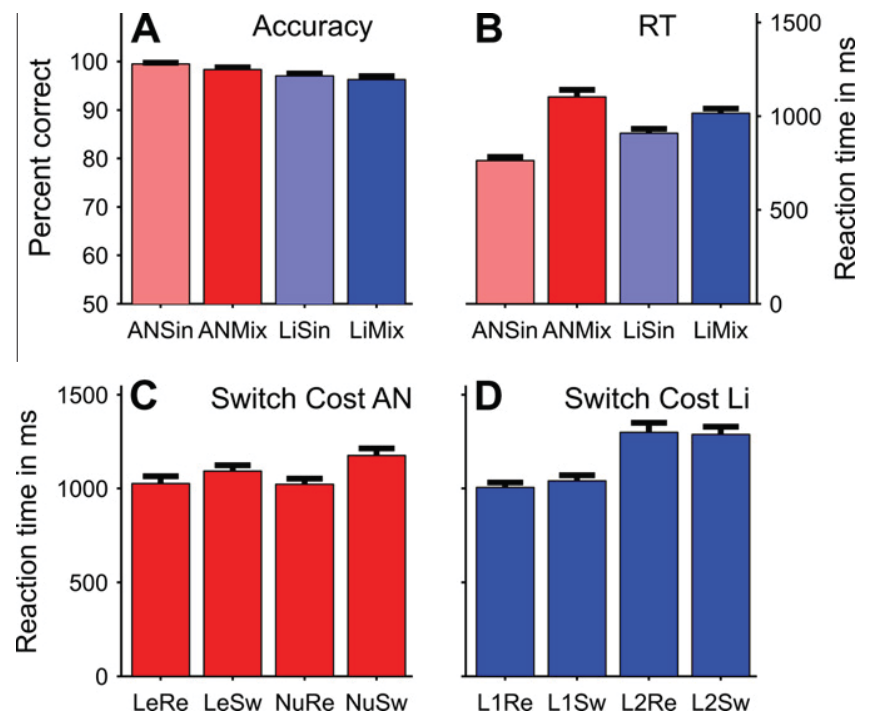

Fig. 2. Behavioural data. Accuracy $(A)$ and reaction time (RT; panel $B$ ) are shown for all four conditions (see text and Fig. 1 for details). Reaction times are also shown as a function of trial type in the mixed context conditions (Switch Cost; panels $C, D$ ). Trial types were either repeat (Re) or switch (Sw). The tasks were letter (Le) or number $(\mathrm{Nu})$ in the alphanumeric condition (AN; panel C), and naming in L1 or L2 in the linguistic conditions ( $\mathrm{Li}$; panel $D$ ).

$p<0.001]$, but no significant main effect of switch status $[F(1,25)=0.30, p=0.590]$, and no significant interaction between language and switch status $[F(1,25)=1.48, p=0.236]$. As Calabria et al. (2012), we also performed correlation analyses on the switch costs (see also Section 4). There was no significant correlation between the alphanumeric (collapsed across task) and the linguistic (collapsed across language) switch costs $(r=0.173, p=0.399)$. There was also no significant correlation between the switch costs for the two "easier" conditions (letter in ANMix and L1 in LiMix: $r=0.063, p=0.760$ ), nor for the two "more difficult" conditions (number in ANMix and L2 in LiMix: $r=-0.085, p=0.679$ ).

\subsection{Behavioural results: analysis of single and mixed contexts}

Mean accuracy was $99.5 \pm 0.2,98.3 \pm 0.5,97.0 \pm 0.5,96.2 \pm 0.7$ for the alphanumeric-single (ANSin), alphanumeric-mixed (ANMix), linguistic-single (LiSin) and linguistic-mixed (LiMix) conditions, respectively (Table 2, Fig. 2A). The reader should note that in this subsection (Section 3.3), the accuracy and reaction time data reported for the mixed conditions refer to only the task/language included in the ERP analyses; that is only the letter or L1 task in ANMix and LiMix conditions, respectively (see Section 2.4). Accuracy was higher in the alphanumeric conditions than in the linguistic conditions. Accuracy was also higher in the single than the mixed conditions. This was confirmed by a repeated-measures ANOVA of accuracy, with domain (alphanumeric, linguistic) and context (single, mixed) as within-participant factors, which revealed significant main effects of domain $[F(1,25)=16.70$,

Table 2

Mean accuracy (\%) and reaction time (RT, in ms), along with the standard error of the mean, as a function of domain and context. Data are only shown for letter and L1 stimuli (see text).

\begin{tabular}{llllll}
\hline \multirow{2}{*}{ Domain } & \multicolumn{2}{l}{ Single-context (Sin) } & & \multicolumn{2}{c}{ Mixed-context (Mix) } \\
\cline { 2 - 3 } \cline { 6 - 7 } & $\%$ & RT (ms) & & $\%$ & RT (ms) \\
\hline Alphanumeric (AN) & $99.5 \pm 0.2$ & $763 \pm 20$ & & $98.3 \pm 0.5$ & $1103 \pm 38$ \\
Linguistic (Li) & $97.0 \pm 0.5$ & $909 \pm 24$ & & $96.2 \pm 0.7$ & $1015 \pm 25$ \\
\hline
\end{tabular}

$p<0.001]$ and context $[F(1,25)=8.37, p=0.008]$, but no significant interaction $[F(1,25)=0.18, p=0.680]$.

Mean reaction times were $763.3 \pm 20,1103.2 \pm 38$; $909.1 \pm 24$, and $1015.6 \pm 25$ for the ANSin, ANMix, LiSin and LiMix conditions, respectively (Table 2, Fig. 2B). Participants took longer to respond during the mixed conditions, than during the single conditions, and this effect was more pronounced for the alphanumeric than for the linguistic conditions. These observations were confirmed by a repeated-measures ANOVA of reaction time, with the within-participant factors of domain and context, which revealed a significant main effect of context $[F(1,26)=174.73, p<0.001]$, as well as interaction $[F(1,25)=63.96, p<0.001]$. There was no significant main effect of domain $[F(1,25)=1.00, p=0.326]$. The difference in reaction times between the single and mixed contexts is referred to as a "mixing" cost (see e.g. Christoffels et al., 2007). The mixing cost is related to, but not equivalent to the "switch cost". The mean reaction time in the mixed-context condition (letter or L1 only) is based on both repeat and switch trials. However, reaction times are greater for the repeat trials in the mixed context than for the single-context condition: 263 (paired t-test: $t(25)=7.09, p<0.001$ ) and $97 \mathrm{~ms}(t(25)=3.28, p=0.003)$ for letter and L1 trials, respectively. This is likely due to the difference between transient and sustained cognitive control (for language, see Christoffels et al., 2007; Wang et al., 2009).

\subsection{ERP waveform analysis: interaction of domain and context}

All four conditions resulted in evoked potential waveforms that are typical for visually presented material, as shown by two exemplar electrodes in Fig. 3A-C. An ANOVA of the ERP data (see Section 2.4) with domain (alphanumeric, linguistic) and context (single, mixed) as within-participant factors revealed a statistically significant interaction $(p<0.05,>20 \mathrm{~ms})$ in both early and late peristimulus time periods (Fig. 3D). The reader is reminded that the ERP waveforms and the pattern of electrode-wise ANOVA results are reference dependent. One of these significant time-periods (approximately 200-250 ms post-stimulus) involved most of the scalp and also coincided with a significant interaction obtained in the source estimation analysis (Fig. 4, see below), which is reference independent. Scalp maps over this time period (Fig. 3E and F) revealed contours which would also suggest an interaction between domain and context. The scalp maps showed bilateral temporal negativities in the linguistic mixed condition, which were not present in any of the other conditions, and an occipital positivity in the linguistic conditions, but not in the alphanumeric conditions. The other periods of a significant interaction were very early (before $100 \mathrm{~ms}$ ) and later (300-400 ms), and both involved the right and posterior scalp quadrants.

\subsection{Source estimation: interaction of domain and context}

LAURA distributed source estimations were first performed over the entire epoch. As with the ERP waveform analysis, a $2 \times 2$ timewise ANOVA with factors of domain and context was performed for each solution point (Figs. 4A and 5C and D). Importantly, this also revealed a time-period (around $200-225 \mathrm{~ms}$ post-stimulus onset) during which there was a significant interaction $(p>0.001$, $>20 \mathrm{~ms}$ ). The interaction was due to modulation of activity (see Section 2.5) in bilateral frontal cortices (inferior, medial, middle, and superior frontal gyri), anterior cingulate, superior temporal cortex and caudate body (Fig. 4A, lower panel). The significant solution points formed two large contiguous clusters (one in each hemisphere) with peaks of significance in left anterior cingulate and right middle frontal gyrus. Post-hoc tests revealed that, within both clusters, this interaction was due to increased source activity 

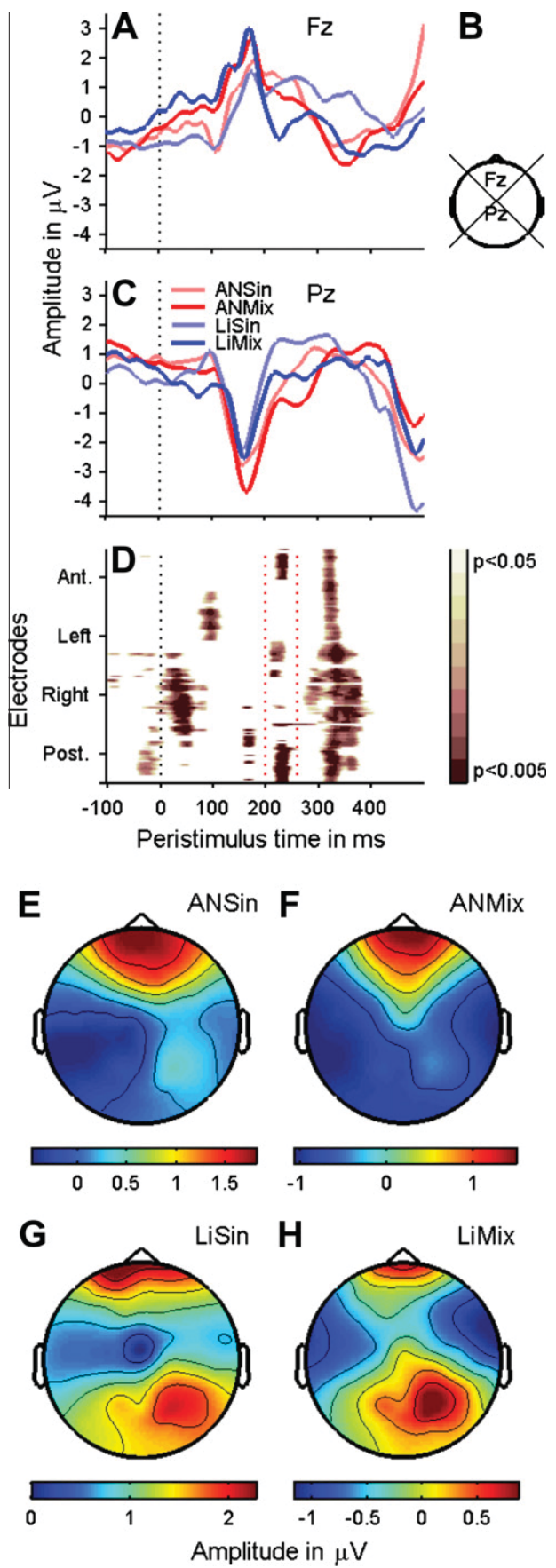

Fig. 3. Grand-average event-related potentials (ERPs: $A$ and $C$ ), interaction in electrode-wise ANOVA (D), and grand-average scalp distribution maps $(E-H)$. ERPs at the electrodes $\mathrm{Fz}(\mathrm{A})$, and $\mathrm{Pz}(\mathrm{C})$ (see $\mathrm{B}$ for location) are shown for all four conditions (see legend in C). D: Coloured regions indicate a significant $(p<0.05$, $>20 \mathrm{~ms}$ ) interaction between domain and context, as a function of electrode and time. For clarity the 128 electrodes have been organised into four groups: anterior ("Ant."), Left, Right, and posterior ("Post."), as approximately shown by the quadrants in B. Black vertical dotted lines show the stimulus onset, and red dotted lines show the time-period (around 200-250 ms post-stimulus) used for the scalp distribution maps of each condition (D-H). ANSin: alphanumeric single; ANMix: alphanumeric mixed, LiSin: linguistic single; LiMix: linguistic mixed.

in the linguistic mixed condition as compared to the other conditions (Fig. 4B and C).

\subsection{Main effects of domain and context}

The ERP and source-estimate ANOVAs revealed significant main effects of domain and context (Fig. 5). The electrode-wise main
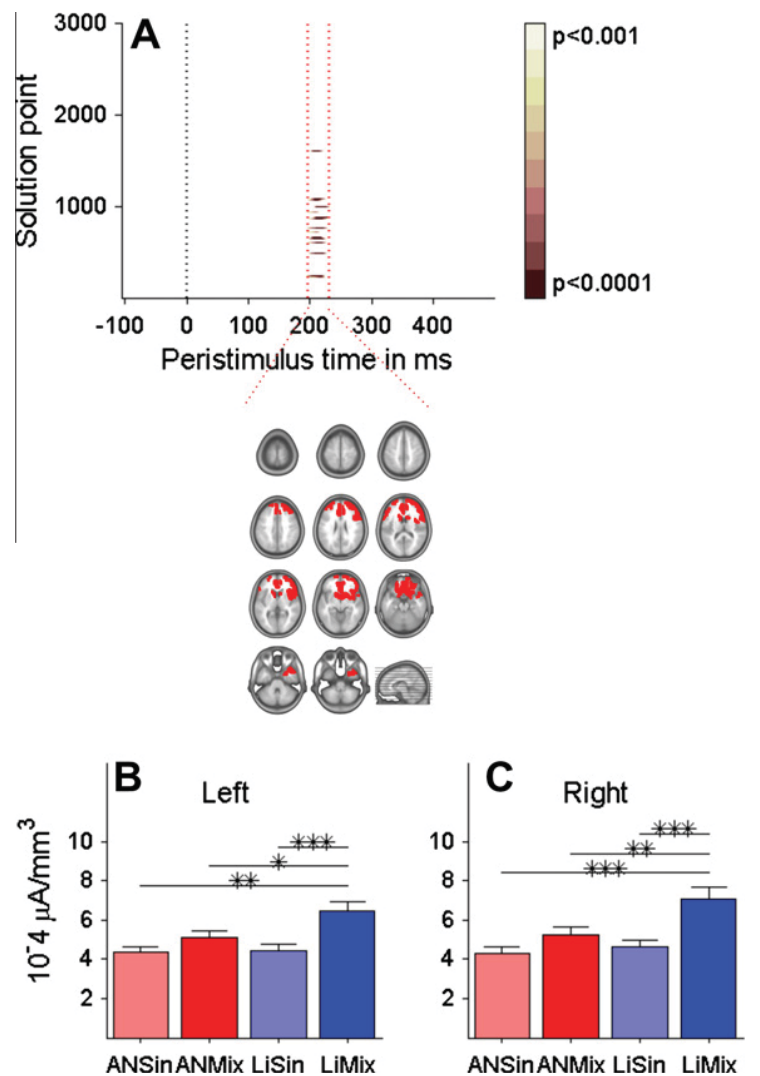

Fig. 4. Interaction between domain and selection in source-estimate ANOVA. (A) In the upper panel, coloured regions indicate a significant $(p<0.001,>20 \mathrm{~ms})$ interaction as a function of solution point and time. The solution points responsible for this interaction over the time window indicated by red dotted vertical lines (see Section 2.5) are shown on axial MRI slices. For these solution points, the averageactivity over the time-window in the left (B) and right (C) hemisphere is shown for all four conditions (labelled as in Fig. 3). Stars mark significant differences (paired $t$ tests, with Bonferroni correction). ${ }^{*} p<0.05 ;{ }^{* *} p<0.01,{ }^{* * *} p<0.001$.

effects were more extensive than the interaction, and for clarity, a more conservative significance threshold was used $(p<0.01$, $20 \mathrm{~ms}$ ). The significance threshold for the source-estimate $(p<0.001,>20 \mathrm{~ms})$ was the same as for the interaction. The main effect of domain begins to be significant very early, even pre-stimulus. This very early period (about -10 to $150 \mathrm{~ms}$ ) was due to modulation of activity in bilateral frontal temporal areas (lower panel of Fig. 5C). Although these bilateral fronto-temporal areas overlapped with the areas found for the interaction (Fig. 4A), they were more extensive, in particular more inferiorly along the temporal cortices. The main effect of context had an early and a late significant time periods. The early period (about $80-180 \mathrm{~ms}$ ) was due to activity in bilateral frontal-temporal areas (lower panel of Fig. 5D), which were even more extensive than for the main effect of domain and the interaction, and included the precentral gyrus. The late period (about 200$400 \mathrm{~ms}$ ) was due not only to activity in frontal-temporal areas, but also inferior parietal and occipital regions.

\section{Discussion}

The current study combined behavioural and electrical neuroimaging (ERPs) to investigate whether, in bilinguals, language control is completely subsidiary to, or partly independent of domain-general cognitive control. On the one hand, both switch-cost analysis of the behavioural data, and a significant interaction in the ANOVA of the ERP data, suggested that the mechanism for bilingual language control is at least partly independent of 

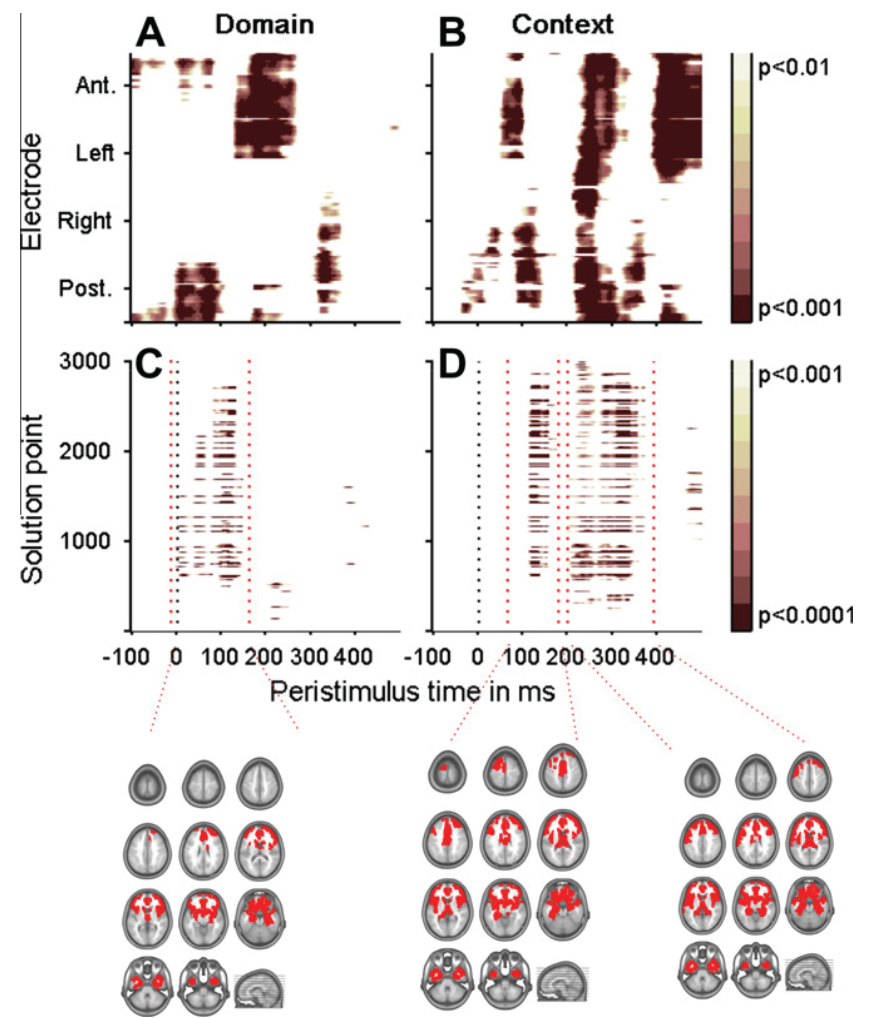

Fig. 5. Main effects of domain (A and $C$ ) and context (B and D) are shown for the ERP ( $A$ and $B$ ) and the source-estimate ANOVAs ( $C$ and D upper panels). Coloured regions indicate statistically significant $(p<0.01 / p<0.001$ for ERP/source estimate, $>20 \mathrm{~ms}$ ) points as a function of time. The lower panels of $C$ and $D$ show the solution points responsible for the main effect (see Section 2.5) over the indicated time window (red dotted vertical lines) displayed on axial MRI slices.

domain-general cognitive control. On the other hand, source estimation revealed that the neuro-anatomical basis of this mechanism is in brain-regions that have previously been associated with both language control and domain-general cognitive control.

Analysis of the behavioural data during the mixed-context conditions showed that although switch costs were asymmetric in the alphanumeric mixed (ANMix) condition, there were negligible and symmetric in the linguistic condition. This result is consistent with Calabria et al. (2012) who found asymmetric and symmetric switch costs in non-linguistic mixed-task and mixed-language conditions, respectively (see Section 1). Some researchers have attributed switch costs to an inhibition mechanism (Green, 1998; Meuter \& Allport, 1999; see however Costa \& Santesteban, 2004; Costa, Santesteban, \& Ivanova, 2006; Verhoef, Roelofs, \& Chwilla, 2009): that is, inhibition is used to suppress the non-target language during a trial. This would lead to a cost of switching target language as inhibition has to be overcome. If there is a difference in proficiency between the two languages, the switch cost should be asymmetric because more inhibition would be required to suppress the stronger language not in use. However, as discussed in Section 1, symmetric switch costs have been found between L1 and a much weaker L3 in early, high-proficient, balanced bilinguals (Calabria et al., 2012; Costa \& Santesteban, 2004; Costa et al., 2006), as well as in unbalanced bilinguals who have daily practice of often switching languages (Christoffels et al., 2007). These previous data suggest that in bilinguals exposed to daily switching, the mechanisms of bilingual language control may be at least partly independent of domain-general cognitive control. In support of this, in both the current and Calabria et al.'s (2012) study, there were no significant correlations of switch costs between linguistic and non-linguistic domains. In the current study, the participants spoke at least two of French, German and English. They were recruited in Geneva, a francophone city which has a significant international anglophone population and is located in the predominantly germanophone nation-state of Switzerland. Therefore, it is highly likely that even the lower proficiency students in this study had a great deal of exposure to language mixing situations in their daily life and this is supported by the finding that the mean difference between in daily exposure time between L1 and L2 was only about $4 \mathrm{~h}$. These data further highlight the importance of considering the ecological context of bilingualism (Green, 2011).

Analysis of the behavioural data for both single- and mixedcontext conditions (letter or L1 only) showed that performance was near ceiling (mean accuracy greater than $96 \%$ ) in all four conditions (ANSin, ANMix, LiSin, and LiMix), and that there was no interaction between domain and context in the ANOVA of the accuracy measures. This suggests that there was little difference in task difficulty across condition. However, there was a statistically significant interaction in the ANOVA of reaction times. This was due to a greater mixing cost in the alphanumeric conditions than in the linguistic conditions, and would thus imply that, if any, the alphanumeric mixed condition was the "most difficult". Therefore, if the significant interaction found in the ERP analysis (see below) were merely due to task difficulty, the alphanumeric mixed condition would be expected to drive the interaction.

The ANOVA of the ERP waveforms revealed a statistically significant interaction between domain and context. This result strongly suggests that language selection and task selection do not rely on the same pattern of brain activity. The finding that the earliest significant interaction is very early $(<100 \mathrm{~ms})$ is consistent with fact that the four experimental conditions were presented in separate blocks. This was necessary in order to prevent the participants from being in "bilingual" mode during the monolingual tasks (Grosjean 1998; Grosjean 2001). However, this means that it is possible that there were differences in brain activity before stimulus presentation, as have been found in many other ERP studies employing blocked designs (for review, see Michel, Koenig, \& Brandeis, 2009). There was a period of significant interaction at around 200-250 ms post stimulus that involved most of the scalp, and also overlapped with the only significant period in the source-estimate ANOVA. This period is thought to correspond with the lemma selection period during picture naming (Levelt, Praamstra, Meyer, Helenius, \& Salmelin, 1998), which would be expected to be a language-specific process. This and later time periods of significant interaction appear to overlap with the N2 component which has been found in previous studies of language control (e.g., Christoffels et al., 2007; Jackson et al., 2001). The N2 is a negative shift over fronto-central sites that has been related to response inhibition (e.g., Jodo \& Kayama, 1992; Thorpe, Fize, \& Marlot, 1996) and conflict monitoring (e.g., Donkers \& Van Boxtel, 2004; Nieuwenhuis, Yeung, Van den Wildenberg, \& Ridderinkhof, 2003). Of note, there was no significant interaction in the last portion of our analysis epoch (400-500 ms). This would suggest that even though the behavioural reaction time data showed a significant interaction, reaction time differences are unlikely to explain the interaction in the ERP data. Before comparing the waveform data with previous research, it is important to remember that the waveforms, and thus statistical analyses, are reference-dependent and that studies may differ in the reference used. Furthermore, differences in experimental paradigm as well as analysis techniques make it difficult to directly compare the current results with previous findings. For example, Khateb et al. (2007) compared the brain responses to picture naming in L1 in two different contexts: a mixed-language context (as in the current study) and a mixed-task context (noun or verb generation). In contrast to the current study, the cue for language or task was presented before the picture, and not simultaneously. Since stimulus-cue interval has been shown to influence 
the dynamics of the brain responses to a language control task (e.g., Verhoef et al., 2009), one may expect diverging findings between the two studies. However, Khateb et al. (2007) found a significant difference between 220 and 300 ms post-cue, which overlaps with the important significant period in the current study. Christoffels et al. (2007) also recorded ERPs during picture naming in singlelanguage and mixed-language conditions. They found that a part of the N2 component (275-375 ms) was sensitive to language context. However their analysis of waveform data was based on two predetermined time-windows, and thus earlier effects could not be ruled out.

The ERP waveform data alone are not sufficient to examine the underlying neural generators of the interaction. At one extreme, the interaction in scalp ERP data could be due to anatomically distinct neural networks for language control, and for domain-general cognitive control (task selection). At the other extreme, domaingeneral cognitive control and language control could rely on anatomically identical networks. If the level of activity within this network is modulated as a function of factors such as cognitive domain (or task difficulty), the current experiment would also result in a significant interaction at the scalp. In order to investigate which of these two options best explained the data, we also performed an ANOVA on the source estimate. This revealed a single period of significant interaction (about 200-225 ms), which overlapped with the ERP waveform interaction, and was due to activity in bilateral, cortical-subcortical, fronto-temporal brain regions. These regions, which were predominantly frontal, included inferior and middle frontal gyrus, caudate, anterior cingulate cortex (ACC), and superior temporal gyri. These regions have previously been implicated in language control in neuroimaging studies, and have also been shown to be involved in general cognitive control (see Section 1). This would strongly suggest that the interaction was primarily due to modulation of activity within anatomically overlapping brain regions responsible for language control and domain-general cognitive control. Most of these previous neuroimaging studies (e.g., Khateb et al., 2007; for review see Luk et al., 2011) compared brain activity during a linguistic task performed in mixed- and single-language contexts. Since both conditions involved the same or similar task (such as picture naming), such a design would not allow direct comparison across cognitive control in different domains. In contrast, the current study employed a $2 \times 2$ factorial analysis to include very different, "non-linguistic" tasks. If there were large brain regions specialised for language control, and not involved in domain-general cognitive control (e.g., task selection), then the design of the current study would be optimal to reveal such regions in the interaction. The current results lend further support to the hypothesis that language selection primarily relies on a network that is within the general cognitive control network. In further support of this, behavioural studies have shown effects of multilingualism on cognitive flexibility and control (for review, see Diamond, 2010). Our findings are also consistent with the recent study by Abutalebi et al. (2011) (see Section 1), who found that in bilinguals, language control and domain-general cognitive control (conflict monitoring) engaged dorsal ACC. That study also included monolingual controls (who had to generate a noun or verb to the same pictures in the mixed-language context), and structural neuroimaging. They showed that bilinguals outperformed monolinguals during the non-linguistic, conflict monitoring task, while showing less ACC activity. Importantly, they showed that for bilinguals, measures of both behavioural and brain-activity in the ACC were positively correlated with local grey matter volume. The authors suggested that long-term practice of two languages could exert a strong influence on neocortical development.

The finding that the interaction was due to bilateral fronto-temporal activity is consistent with previous studies which have shown involvement of right-hemisphere regions in language control (for review, see Luk et al., 2011). The peak of the interaction within the left hemisphere was in the ACC. As discussed above, the ACC has been proposed as a crucial node for language control on the basis of clinical and neuroimaging evidence (for review, see Abutalebi \& Green, 2008). The peak of the interaction within the right hemisphere was in the right middle frontal gyrus, which has also previously been implicated in language control (Hernandez, Dapretto, Mazziotta, \& Bookheimer, 2001). Post-hoc test showed that in both the left- and right-hemisphere clusters, the interaction was due to significantly greater activity during the linguistic mixed condition, compared with the other three conditions. It seems unlikely that this was simply due to the linguistic mixed condition being the "most difficult", as this was not supported by analysis of the behavioural data.

Analysis of the ERP waveforms and source estimates also resulted in significant main effects of domain and selection, although these were not of primary interest in the current study. The main effect of domain can be attributed to the fact that the linguistic and alphanumeric conditions differed in stimuli and in task. This has been found previously (for review, see Michel et al., 2009) and will not further be discussed here. The main effect of context showed an early (around $100 \mathrm{~ms}$; predominantly frontal-temporal) and a late (200-400 ms; frontal-temporal and some parietal and occipital regions) effect. Some of these effects of context may be due to differences in potential eye movement preparation between the single (stimulus always above or below the horizontal meridian) and mixed conditions (stimulus could be above or below). Interestingly, many previous studies have also implicated some parts of the parietal lobe (e.g., supramarginal gyrus) in language control. In the current study, parietal regions showed a main effect of context, but not an interaction, which would imply that they were equally involved in both task and language selection.

Taken together, the switch-cost differences in the behavioural data, and the interaction in the ERP waveform data, strongly suggest that there is a difference in processing mechanism between language control and domain-general cognitive control. This would mean that an important component of language control is at least, partly independent of domain-general cognitive control. Source estimate analysis revealed that the neuro-anatomical basis for this partly-independent language mechanism is located in brain regions previously associated with domain-general cognitive control. The results suggest that in bilinguals confronted with switching on a daily basis, the mechanism of neural processing underlying language control differs from domain-general cognitive control, yet the neuro-anatomical basis of cognitive control in these two domains remains the same.

Future studies may further explore whether the current results are related to the plasticity of cognitive control mechanisms, on different time-scales. On a short time-scale, the current study was not able to replicate the finding of Calabria et al. (2012) that the direction of switch cost asymmetry changed across experimental blocks for the mixed-language, but not the mixed-task condition. On a longer time-scale, participants in the current study had several years of daily practice in mixed-language conditions, but not of alphanumeric tasks. What pattern of behavioural and neuroimaging results would be obtained if participants were exposed to the non-linguistic mixed-task context on a daily basis for a prolonged period? The fMRI study of Abutalebi et al. (2011) suggests that long-term bilingualism leads to changes in structure and function of the ACC. In contrast to the current study, their results suggest that these changes are relevant for cognitive control of non-linguistic tasks. However, it is difficult to directly compare the two studies due to differences in the behavioural paradigms and neuroimaging approach. It would be important to perform the current study in an fMRI setting, which offers superior spatial resolution. 


\section{Acknowledgments}

This work was supported by a Grant from the Swiss National Science Foundation to JMA (\#32-138497, \#325100-118362). The Cartool software (sites.google.com/site/fbmlab/Cartool.htm) has been programmed by Denis Brunet, from the Functional Brain Mapping Laboratory, Geneva, Switzerland, and was supported by the Centre for Biomedical Imaging of Geneva and Lausanne. We would like to thank Jean-François Knebel (University of Lausanne) for the software to carry out the ANOVAs.

\section{References}

Abutalebi, J., Annoni, J. M., Zimine, I., Pegna, A. J., Seghier, M. L., Hannelore, L. J., et al. (2008). Language control and lexical competition in bilinguals: An event-related fMRI study. Cerebral Cortex, 18, 1496-1505.

Abutalebi, J., Della Rosa, P. A., Green, D. W., Hernández, M., Scifo, P., et al. (2011). Bilingualism tunes the anterior cingulate cortex for conflict monitoring. Cerebral Cortex. http://dx.doi.org/10.1093/cercor/bhr287.

Abutalebi, J., Della Rosa, P. A., Tettamanti, M., Green, D. W., \& Cappa, S. F. (2009). Bilingual aphasia and language control: A follow-up fMRI and intrinsic connectivity study. Brain and Language, 109, 141-156.

Abutalebi, J., \& Green, D. (2008). Control mechanisms in bilingual language production: Neural evidence from language switching studies. Language and Cognitive Processes, 23, 557-582.

Abutalebi, J., Miozzo, A., \& Cappa, S. F. (2000). Do subcortical structures control language selection in bilinguals? Evidence from pathological language mixing. Neurocase, 6, 101-106.

Aglioti, S., \& Fabbro, F. (1993). Paradoxical selective recovery in a bilingual aphasic following subcortical lesion. Neuroreport, 4, 1359-1362.

Alario, F. X., \& Ferrand, L. (1999). A set of 400 pictures standardized for French: Norms for name agreement, image agreement, familiarity, visual complexity, image variability, and age of acquisition. Behavior Research Methods, Instruments, and Computers, 31, 531-552.

Brunet, D., Murray, M., \& Michel, C. M. (2011). Spatiotemporal analysis of multichannel EEG: CARTOOL. Computational Intelligence and Neuroscience. http://dx.doi.org/10.1155/2011/813870.

Calabria, M., Hernández, M., Branzi, F. M., \& Costa, A. (2012). Qualitative differences between bilingual language control and executive control: Evidence from taskswitching. Frontiers in Psychology. 103389/psyg.2011.00399.

Christoffels, I. K., Firk, C., \& Schiller, N. O. (2007). Bilingual language control: An event-related brain potential study. Brain Research, 1147, 192-208.

Costa, A., \& Santesteban, M. (2004). Lexical access in bilingual speech production: Evidence from language switching in highly proficient bilinguals and L2 learners. Journal of Memory and Language, 50, 491-511.

Costa, A., Santesteban, M., \& Ivanova, I. (2006). How do highly proficient bilinguals control their lexicalization process? Inhibitory and language-specific selection mechanisms are both functional. Journal of Experimental Psychology: Learning, Memory and Cognition, 32, 1057-1074.

Delorme, A., \& Makeig, S. (2004). EEGLAB: An open source toolbox for analysis of single-trial EEG dynamics including independent component analysis. Journal of Neuroscience Methods, 134, 9-21.

Diamond, J. (2010). The benefits of multilingualism. Science, 330, 332-333.

Donkers, F. C., \& Van Boxtel, G. J. (2004). The N2 in go/no-go tasks reflects conflict monitoring and not response inhibition. Brain and Cognition, 56, 165-176.

Dove, A., Pollmann, S., Schubert, T., Wiggins, C. J., \& von Cramon, D. Y. (2000). Prefrontal cortex activation in task switching: An event-related fMRI study. Cognitive Brain Research, 9, 103-109.

Grave de Peralta, R., Gonzalez Andino, S., \& Gomez Gonzalez, C. M. (2004). The biophysical foundations of the localisation of encephalogram generators in the brain. The application of a distribution type of model to the localisation of epileptic foci. Revista de Neurologia, 39, 748-756.

Grave de Peralta, R., Gonzalez Andino, S., Lantz, G., Michel, C. M., \& Landis, T. (2001). Noninvasive localization of electromagnetic epileptic activity. I. Method descriptions and simulations. Brain Topography, 14, 131-137.

Green, D. W. (1998). Mental control of the bilingual lexico-semantic system. Bilingualism: Language and Cognition, 4, 169-178.

Green, D. W. (2011). Language control in different contexts: The behavioural ecology of bilingual speakers. Frontiers in psychology. http://dx.doi.org/10.3389/ fpsyg.2011.00103.
Grosjean, F. (1998). Studying bilinguals: Methodological and conceptual issues. Bilingualism Language and Cognition, 1, 131-141.

Grosjean, F. (2001). The bilingual's language modes. In L. Janet (Ed.), One mind, two languages: Bilingual sentence processing (pp. 1-22). Oxford: Blackwell.

Hernandez, A. E., Dapretto, M., Mazziotta, J., \& Bookheimer, S. (2001). Language switching and language representation in Spanish-English bilinguals: An fMRI study. NeuroImage, $14,510-520$.

Hervais-Adelman, A. G., Moser-Mercer, B., \& Golestani, N. (2011). Executive control of language in the bilingual brain: Integrating the evidence from neuroimaging to neuropsychology. Frontiers in Psychology, 2. http://dx.doi.org/10.3389/ fpsyg.2011.00234.

Jackson, G. M., Swainson, R., Mullin, A., Cunnington, R., \& Jackson, S. R. (2001). ERP correlates of executive control during repeated language switching. Bilingualism Language and Cognition, 4, 169-178.

Jackson, G. M., Swainson, R., Mullin, A., Cunnington, R., \& Jackson, S. R. (2004). ERP correlates of a receptive language-switching task. The Quarterly Journal of Experimental Psychology A: Human experimental psychology, 57, 223-240.

Jodo, E., \& Kayama, Y. (1992). Relation of a negative ERP component to response inhibition in a Go/No-go task. Electroencephalography and Clinical Neurophysiology, 82, 477-482.

Khateb, A., Abutalebi, J., Michel, C. M., Pegna, A. J., Lee-Jahnke, H., \& Annoni, J. M. (2007). Language selection in bilinguals: A spatio-temporal analysis of electrical brain activity. International Journal of Psychophysiology, 65, 201-213.

Kho, K. H., Duffau, H., Gatignon, P., Leijten, F. S. S., Ramsey, N. F., van Rijen, P. C., et al. (2007). Involuntary language switching in two bilingual patients during the Wada test and intraoperative electrocortical stimulation. Brain and Language, 101, 31-37.

Kimberg, D. Y., Aguirre, G. K., \& D'Esposito, M. (2000). Modulation of task-related neural activity in task-switching: An fMRI study. Cognitive Brain Research, 10, 189-196.

Levelt, W. J., Praamstra, P., Meyer, A. S., Helenius, P., \& Salmelin, R. (1998). An MEG study of picture naming. Journal of Cognitive Neuroscience, 10, 553-567.

Luk, G., Green, D. W., Abutalebi, J., \& Grady, C. (2011). Cognitive control for language switching in bilinguals: A quantitative meta-analysis of functional neuroimaging studies. Language and Cognitive Processes. http://dx.doi.org/ 10.1080/01690965.2011.613209.

Metz-Lutz, M. N., Kremin, H., Deloche, G., Hannequin, D., Ferrand, I., Perrier, D., et al. (1991). Standardisation d'un test de dénomination orale: Contrôle des effets de l'âge, du sexe et du niveau de scolarité chez des sujets adultes normaux (Standardization of an oral naming test controlling the effects of age, gender and education level in normal adult subjects). Revue de Neuropsychologie, 1, 73-95.

Meuter, R. F. I., \& Allport, A. (1999). Bilingual language switching in naming: Asymmetrical costs of language selection. Journal of Memory and Language, 40, 25-40.

Michel, C. M., Koenig, T., \& Brandeis, D. (2009). Electrical neuroimaging in the time domain. In C. M. Michel, T. Koenig, D. Brandeis, L. R. R. Giannotti, \& J. Wackermann (Eds.), Electrical neuroimaging (pp. 111-144). Cambridge: Cambridge University Press.

Moritz-Gasser, S., \& Duffau, H. (2009). Cognitive processes and neural basis of language switching: Proposal of a new model. NeuroReport, 20, 1577-1580.

Nieuwenhuis, S., Yeung, N., Van den Wildenberg, W., \& Ridderinkhof, K. R. (2003). Electrophysiological correlates of anterior cingulate function in a go/no go task: Effects of response conflict and trial type frequency. Cognitive, Affective, and Behavioral Neuroscience, 3, 17-26.

Oldfield, R. C. (1971). The assessment and analysis of handedness: The Edinburgh inventory. Neuropsychologia, 9, 97-113.

Price, C. J., Green, D. W., \& Von Sutdnitz, R. (1999). A functional imaging study of translation and language switching. Brain, 122, 2221-2235.

Rogers, R., \& Monsell, S. (1995). Costs of a predictable switch between simple cognitive tasks. Journal of Experimental Psychology, 124, 207-231.

Thorpe, S., Fize, D., \& Marlot, C. (1996). Speed of processing in the human visual system. Nature, 381, 520-522.

Verhoef, K., Roelofs, A., \& Chwilla, D. J. (2009). Role of inhibition in language switching: Evidence from event-related potentials in overt picture naming. Cognition, 110, 84-99.

Wang, Y., Kuhl, P. K., Chen, C., \& Dong, Q. (2009). Sustained and transient language control in the bilingual brain. NeuroImage, 47, 414-422.

Wartenburger, I., Heekeren, H. R., Abutalebi, J., Cappa, S., Villringer, A., \& Perani, D. (2003). Early setting of grammatical processing in the bilingual brain. Neuron, $37,159-170$.

Ye, Z., \& Zhou, X. (2009). Executive control in language processing. Neuroscience and Biobehavioral Reviews, 33, 1168-1177. 